\title{
Les coraux de l'océan Pacifique et l'enregistrement des événements El Niño
}

Anne Juillet-Leclerc

\section{OpenEdition}

1 Journals

Édition électronique

URL : https://journals.openedition.org/jso/4932

DOI : $10.4000 /$ jso.4932

ISSN : $1760-7256$

Éditeur

Société des océanistes

\section{Édition imprimée}

Date de publication : 15 décembre 2008

Pagination : 11-24

ISBN : 978-2-85430-012-3

ISSN : 0300-953x

\section{Référence électronique}

Anne Juillet-Leclerc, "Les coraux de l'océan Pacifique et l'enregistrement des événements El Niño », Journal de la Société des Océanistes [En ligne], 126-127 | Année 2008, mis en ligne le 15 décembre 2011, consulté le 21 septembre 2021. URL : http://journals.openedition.org/jso/4932 ; DOI : https:// doi.org/10.4000/jso.4932

\section{(c) $($ $९ \ominus$}

Journal de la société des océanistes est mis à disposition selon les termes de la Licence Creative Commons Attribution - Pas d'Utilisation Commerciale - Pas de Modification 4.0 International. 


\section{Les coraux de l'océan Pacifique et l'enregistrement des événements El Niño}

par

Anne JUILLET-LECLERC ${ }^{*}$

\section{RÉSUMÉ}

Les coraux tropicaux gardent en mémoire, dans leur squelette, les conditions climatiques comme la température de l'océan, l'abondance des précipitations ou l'ensoleillement qu'ils ont connus, il y a quelques dizaines d'années, voire quelques siècles. Mais ces informations sont cryptées par l'organisme et seules des technologies adaptées nous permettent une traduction fidèle, en termes météorologiques et océanographiques, de données physico-chimiques, notamment isotopiques, mesurables dans les coraux. Et nous n'en sommes qu'aux débuts de ces types de déchiffrage.

MoTS-CLÉS : corail, squelette, biominéralisation, climat tropical, El Niño, reconstitution climatique

Pour la plupart d'entre nous, les coraux sont associés à des fonds sous-marins luxuriants où les extrémités de leurs bras ou les bords de leurs tissus flottent au gré de la houle, mi-animaux, mi-fleurs. Leurs couleurs varient du vert tendre au violet profond et rivalisent avec le rouge ou l'indigo des gorgones et des anémones de mer. Dans ce paysage tout à la fois changeant et immobile se faufilent d'innombrables poissons, gros et petits, aux nageoires d'un jaune ou d'un bleu improbable. Cette vie exubérante nous fait immanquablement penser à une oasis, et ce rapprochement n'est pas totalement sans raison, car les coraux sont des organismes pionniers qui colonisent les zones les plus pauvres du monde océanique et les transforment en des univers foi-

\begin{abstract}
Informations on past climatic conditions such as sea surface temperature, abundance of precipitations or solar irradiation, during the last decades, or even centuries, are captured in coral skeletons. However, these informations are strongly impacted by biology and we need selected technologies to convert dependably chemical, and especially isotopic values, into environmental atmospheric and oceanographic data.
\end{abstract}

KEYwORDS: coral, skeleton, biomineralization, tropical climate, El Niño, climate reconstruction

sonnant de vie. Le corail appréciant des températures supérieures à $18^{\circ} \mathrm{C}$, on le trouve donc surtout dans les zones intertropicales. En revanche, il est très sensible aux excès de température et le réchauffement global actuel, dû à l'effet de serre du $\mathrm{CO}_{2}$ atmosphérique, met en réel péril l'équilibre et la diversité biologiques des milieux récifaux dès que la température des eaux océaniques superficielles s'élève et dépasse 28 ou $29^{\circ} \mathrm{C}$.

\section{Le corail : animal, végétal et minéral}

Le corail est un animal de petite taille qui vit le plus souvent en colonies et forme un squelette

\footnotetext{
* Laboratoire des Sciences du climat et de l'environnement, Gif-sur-Yvette, Anne.Juillet-Leclerc@1sce.ipsl.fr
} 
Septe

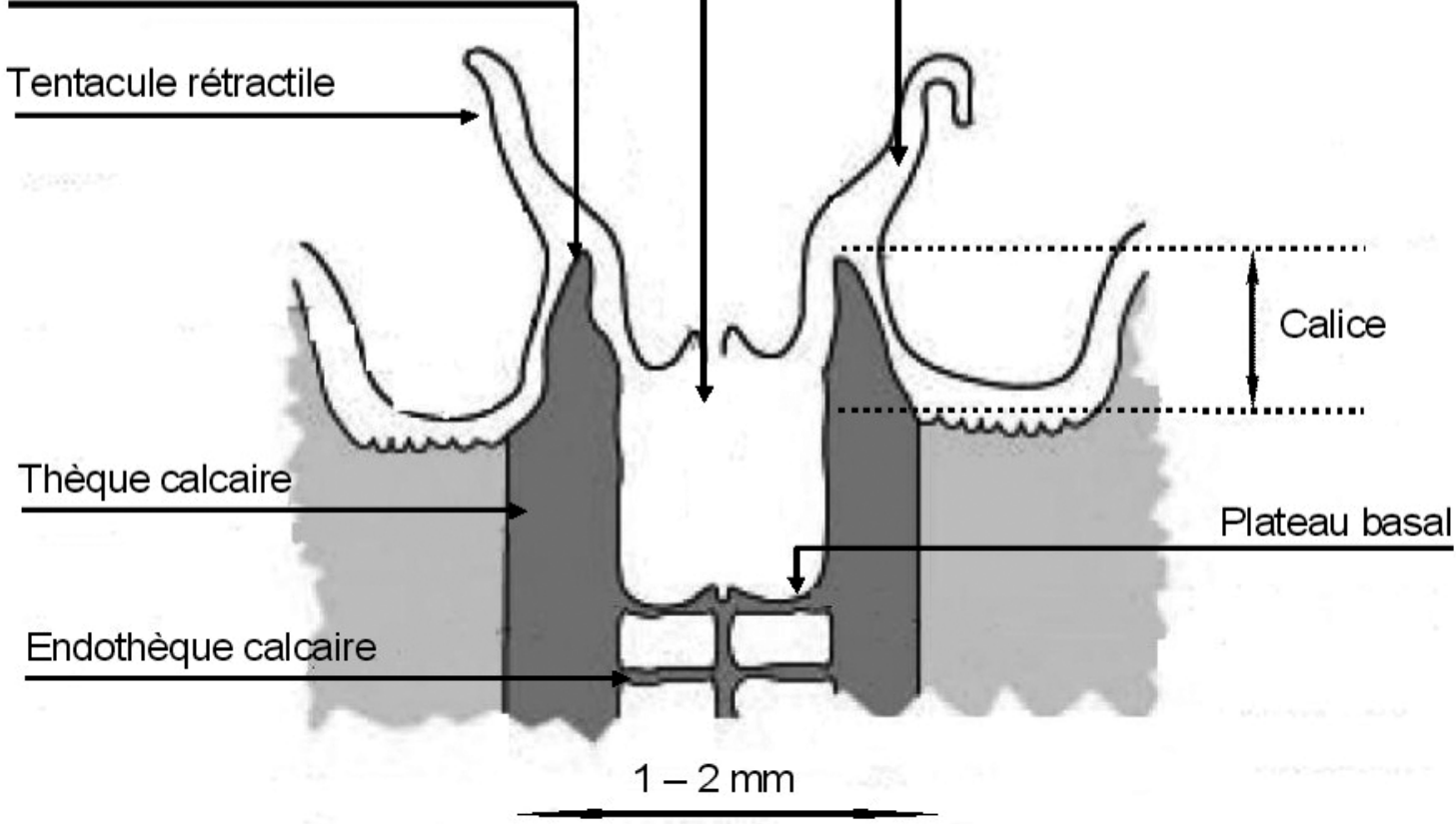

Polype

FiguRE 1. - Vue schématique de l'organisation d'un polype corallien. Les tissus mous sont en blanc, le squelette en gris sombre. Les algues symbiotiques, assurant la fonction chlorophyllienne, sont localisées dans les tissus mous superficiels (http://www.centrescientifique.mc)

d'aragonite $^{1}$. Au contraire des os humains, ce squelette est externe par rapport à la partie proprement organique de l'animal, comme le montre le schéma de la figure 1. Bien que biologiquement assez simple, cet organisme vit en symbiose avec des micro-algues photosynthétiques et colorées (les zooxanthelles) qui se trouvent dans les appendices et les tissus les plus externes de l'animal (figure 1). Ce sont elles qui contiennent les pigments brillants qui attirent la vue dans les eaux tropicales. L'expulsion de ces végétaux provoque le blanchissement du corail sous l'effet du stress que ressent l'animal lorsque la température de l'eau océanique superficielle devient excessive pour lui.

Les colonies peuvent prendre des apparences multiples, par exemple branchues évoquant la ramure d'un arbuste ou d'un cerf ou plus massives. Dans ce dernier cas, la colonie corallienne peut apparaître comme une énorme boule (figure 2) constituée de milliers de polypes (nom désignant un animal individuel), tous d'un même clone. Ces «boules » peuvent atteindre trois à quatre mètres de diamètre et résultent $\mathrm{du}$ développement successif de nombreux polypes.
Elles peuvent s'édifier au cours de plusieurs siècles, au cours desquels l'animal et ses algues symbiontes auront toujours occupé la superficie de la colonie (Robin et al., 1987).

Le diamètre de la colonie massive est d'environ $4 \mathrm{~m}$ et correspond à une durée de croissance d'environ quatre siècles. La partie vivante est localisée dans les quelques millimètres supérieurs de la colonie.

Le carbonate de calcium (calcaire) dont est constitué le squelette corallien est formé d'atomes de calcium, d'oxygène et de carbone. Nous rappellerons, dans la suite (voir « Les indicateurs paléoclimatiques »), que les atomes d'oxygène contenus dans ces minéraux peuvent garder des traits de composition, dits isotopiques, qui ont été déterminés par la température ayant régné lors de leur dépôt. Les atomes d'oxygène sont, en effet, dans la nature, essentiellement des atomes ${ }^{16} \mathrm{O}$ et ${ }^{18} \mathrm{O}$, appelés des isotopes, dont les masses sont, respectivement, de 16 et de 18 unités de masse molaire. Ces atomes n'ont donc pas tous le même poids. Or la quantité relative des atomes lourds $\left({ }^{18} \mathrm{O}\right)$ et légers $\left({ }^{16} \mathrm{O}\right)$ dans l'aragonite se révèle différente en fonction de la température de 


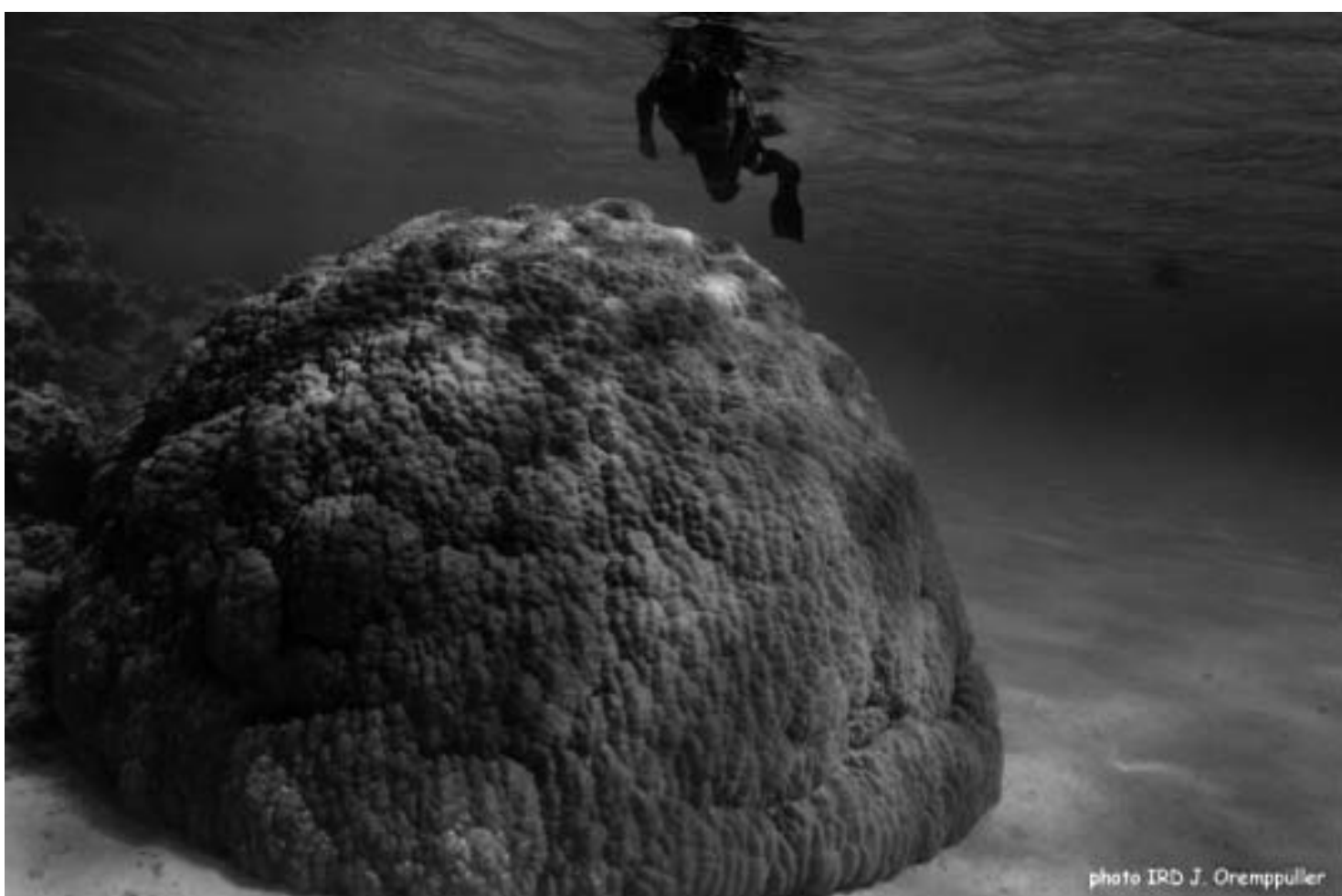

FIgure 2. - Colonie de Porites

formation du minéral. Le rapport du nombre d'atomes lourds et d'atomes légers, dénommé « rapport isotopique de l'oxygène ${ }^{2}$ » est donc un thermomètre isotopique. Au milieu du siècle dernier, en même temps que ce résultat géochimique fondamental, s'élaborèrent les spectromètres de masse, appareils permettant de mesurer les quantités des isotopes des éléments chimiques et, notamment, celles de l'oxygène dans des échantillons calcaires.

Le squelette de la colonie de Porites représenté dans la figure 2, qui ressemble à un énorme chou-fleur, contient donc des indicateurs chimiques reflétant la température de l'eau dans laquelle chacun des minéraux qui le constituent a cristallisé. Sachant que les coraux de l'espèce photographiée sont capables d'ajouter à leur squelette une épaisseur de plus de $1 \mathrm{~cm}$ de minéral au cours d'une année, l'on peut considérer qu'une colonie corallienne de trois à quatre mètres de diamètre a enregistré les variations de la température au cours de plus de trois siècles, avec un détail temporel de l'ordre du mois. Si aucun changement défavorable à la croissance des polypes n'intervient, le squelette est secrété par le corail en continu et toutes les variations thermiques qui se sont succédé dans son environ- nement au cours de sa croissance sont inscrites dans ses minéraux aragonitiques.

Le couple constitué par la succession d'une bande claire et d'une bande sombre correspond à un dépôt annuel. Mais l'on ne sait pas associer la densité d'une couche à un paramètre donné de l'environnement.

Si l'on effectue un carottage ${ }^{3}$ dans cette colonie, il est possible d'apercevoir, même à l'œil nu, des anneaux concentriques, qui correspondent à des densités variables d'aragonite (figure 3 ). Une année de développement se traduit par l'alternance d'une bande claire (peu dense) et d'une bande plus foncée (plus dense), témoins respectifs des deux saisons annuelles que connaissent les zones intertropicales. Ces couches apparaissent encore plus nettement sur une radio aux rayons X (figure 3). Les variations de température peuvent donc aisément être datées, à condition de connaître la date de formation de la couche de surface de la colonie et de décompter le nombre de couches séparant celle-ci de la couche concernée. Il faut préciser que, si la valeur isotopique mesurée dépend de la température, elle dépend aussi de la composition isotopique de l'oxygène de l'eau de mer où s'est formé le minéral, composition qui varie, elle-même, comme la salinité.

2. Quantité d'isotopes lourds/isotopes légers, par exemple pour l'oxygène ${ }^{18} \mathrm{O} /{ }^{16} \mathrm{O}$.

3. Le prélèvement d'un cylindre d'aragonite parallèlement à la direction de croissance du corail. 


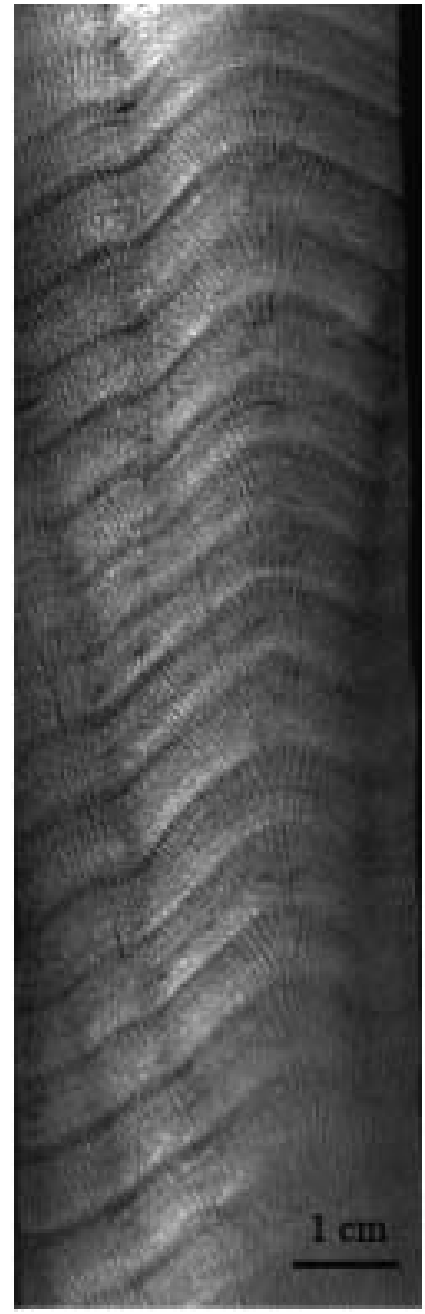

FIgURE 3. - Radiographie X d'une plaquette taillée dans le squelette d' une colonie de Porites

Ainsi le squelette du corail contient-il des informations sur la température et la salinité ayant varié de façon continue au cours de la vie de l'animal. Les variations successives de ces paramètres reflètent donc celles de l'« environnement » dans lequel a vécu cet animal. Mais quel intérêt y a-t-il à connaître la température du passé ?

\section{El Niño : le changement climatique majeur à échelle humaine}

Nous avons noté que le corail symbiotique ${ }^{4}$ se développe dans des eaux plutôt chaudes, autour et dans la ceinture tropicale des océans Atlantique, Pacifique et Indien. La variation climatique majeure qui se produit à l'échelle de la vie humaine, El Niño, est très spectaculaire dans l'océan Pacifique intertropical. C'est, en effet, dans cette zone que l'eau de mer est la plus chaude (figure 4) et, pour cette raison, que s'y forment des précipitations abondantes qui alimentent ensuite en pluies les zones de plus hautes latitudes. Ce phénomène représente le transfert d'énergie atmosphérique le plus important à l'échelle du globe. En effet, tout comme l'évaporation assure, au cours de la transpiration, un rafraîchissement corporel, l'évaporation de la couche superficielle de l'océan tropical y provoque localement une baisse de chaleur latente. L'énergie accumulée dans les molécules chaudes au sein de la vapeur d'eau sera restituée au cours des précipitations qui interviendront à des latitudes supérieures. La zone intertropicale océanique est donc une zone cardinale du système climatique global. Ces échanges naturels d'eau et d'énergie se superposent maintenant à ceux qu'engendrent diverses activités humaines, notamment la libération dans l'atmosphère de quantités considérables de gaz carbonique ${ }^{5}$. Notre intention étant de reconstituer les variations climatiques naturelles dans l'océan Pacifique, afin de reconnaître leur place et leur part dans les changements climatiques actuels, il nous faut les identifier, ainsi que leurs effets, dans des périodes où l'impact « additionnel » de l'homme sur l'atmosphère puisse être négligé. Ces périodes sont celles qui ont précédé la révolution industrielle.

Pendant une période "normale" les eaux chaudes sont localisées dans le Pacifique occidental, alors qu'elles s'accumulent dans l'océan Pacifique central et oriental pendant les périodes d'El Nino. La limite entre les eaux chaudes superficielles et des eaux plus froides, sousjacentes, dans l'océan oriental, désignée sous le nom de thermocline, est alors plus profonde que pendant une période «normale». Les nuages sont, alors également, abondants au dessus du Pacifique occidental (http://www.cdc.noaa.gov/ ENSO).

\section{Une interaction entre l'océan et l'atmosphère}

Comme nous l'observons dans la figure 4, El Niño affecte à la fois l'atmosphère et l'océan, sans que l'on comprenne clairement où se trouve le mécanisme précurseur. En période « normale », des alizés forts soufflent des côtes américaines en direction de l'Indonésie, les pré-

4. Qui vit avec une algue symbionte.

5. Ce carbone était stocké depuis des millions d'années sous forme fossile : charbons, pétroles. 


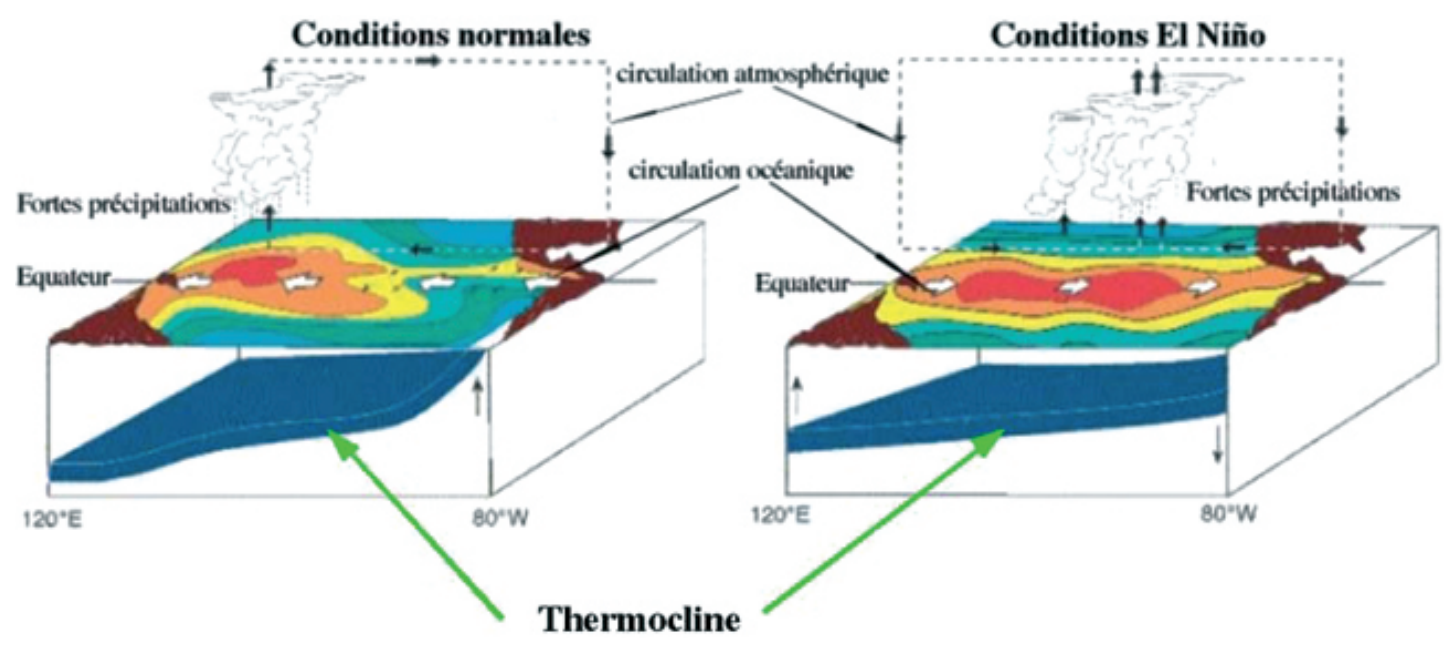

FiguRE 4. - Enregistrement de changements océaniques et atmosphériques pendant une période normale et pendant un événement El Niño, dans l'océan Pacifique tropical. Les températures les plus élevées, à la surface de la mer, correspondent aux zones rouges (voir cahier couleur après p. 144)

occupations sont abondantes dans la zone Ouest $\mathrm{du}$ Pacifique intertropical, en particulier au-dessus de la partie la plus chaude de cet océan - et de tous les océans - appelée, en anglais, la Warm Pool (l'eau de surface de l'océan peut y atteindre 29 à $30^{\circ} \mathrm{C}$ tout au long de l'année). Pendant ce temps, le long de la côte tropicale de l'Amérique du Sud, à l'Est du Pacifique et le long de l'Équateur, des eaux froides venues de quelques centaines de mètres de profondeur atteignent la surface dans un mouvement d'upwelling. Ces eaux, riches en éléments nutritifs, constituent le lieu privilégié du développement de grandes quantités de poissons. Tel est le cas des eaux longeant les côtes du Pérou. Si les alizés qui repoussent les eaux chaudes sur la partie ouest du bassin faiblissent, la masse d'eau accumulée du côté indonésien reflue sur les côtes américaines et recouvre les eaux froides et riches qui les bordaient. Les précipitations sont alors redistribuées et deviennent particulièrement abondantes au droit de zones habituellement désertiques, en particulier sur les pentes occidentales de la chaîne andine où elles déclenchent d'énormes coulées de boue. Simultanément, les zones qui étaient normalement bien arrosées, en Indonésie ou en Australie, sont soumises à la sécheresse. Le nom de cet événement, El Niño, fait référence à la période de Noël. En effet, les conquistadors espagnols furent les premiers à décrire les dégâts causés par ce phénomène sur le nouveau continent. Comme celui-ci se manifestait en Décembre, juste avant l'arrivée de l'enfant Jésus, ils l'appelèrent El Niño. Il est normale- ment suivi de conditions dites «normales», températures plus froides, précipitations plus abondantes localisées à l'ouest du domaine Pacifique : c'est La Niña.

\section{Les conséquences d'El Niño}

El Niño, aussi appelé ENSO ${ }^{6}$, a commencé à susciter la curiosité à l'échelle internationale lorsque ses conséquences provoquèrent une crise économique sans précédent au Pérou au début des années 1970. En effet, durant les années précédentes, ce pays avait développé une économie basée sur la pêche et la mise de poissons en conserve. En 1972, les eaux fertiles de la bordure pacifique furent recouvertes d'eaux plus chaudes venant de l'ouest du Pacifique, ce qui inhiba la remontée des eaux froides orientales et, partant, le développement des poissons. S'en suivit une crise économique aiguë. Depuis, des efforts ont été faits pour essayer de comprendre le mécanisme de la genèse de ce phénomène afin d'en prédire la manifestation. Cependant, jusqu'à maintenant, ces efforts n'ont guère été couronnés de succès. C'est ainsi qu'en 1997, les services météorologiques américains ont tardé à reconnaître les prémices d'El Niño. Les signes avantcoureurs ne ressemblaient pas tout à fait aux observations réalisées lors d'épisodes précédents et les anomalies se succédaient selon un ordre inhabituel. Les eaux chaudes sont arrivées au large des côtes sud-américaines en juin-juillet 1997 et non en décembre 1997. Les avertisse-

6. El Niño-Southern Oscillation (ENSO) associe un phénomène océanique (El Niño) et un phénomène atmosphérique (Southern Oscillation ou oscillation climatique de l'hémisphère Sud). 
ments des météorologues se sont sans doute fait attendre mais, malgré un retard de quelques mois, ces avertissements auraient pu être pris en compte par les gouvernements des pays bordant la côte sud-américaine et conduire à des recommandations pratiques, par exemple planter du riz plutôt que du coton et protéger les infrastructure... Mais ceux-ci n'ont pas cru à des signaux atypiques. L'épisode El Niño qui se produisit de 1997 à 1998 fut l'événement « environnemental » majeur $\mathrm{du} \mathrm{xx}^{\mathrm{e}}$ siècle qui s'achevait. Les pertes économiques ont largement dépassé les sommes astronomiques atteintes en 1982, plus de 8 milliards de dollars. La sécheresse enregistrée à l'ouest du Pacifique en juin 1997 fut telle que la mousson d'été a tardé à débuter et que les feux allumés en fin de récolte, comme chaque année, par les Indonésiens, n'ont pu être éteints comme à l'habitude par la mousson d'été. Ces feux ont dévasté des forêts primaires à Bornéo et un énorme nuage de fumée a obscurci le ciel des pays voisins pendant plusieurs mois. Les conséquences d'El Niño ne sont pas restreintes à l'océan Pacifique tropical, elles affectent également, profondément, l'océan Indien voisin. Elles ont contribué aux fluctuations du débit du Nil au cours des siècles passés. Les caractéristiques des masses d'eau de l'Atlantique Sud sont aussi modifiées. Même les variations des précipitations de l'ouest de l'Europe semblent en garder les empreintes. De plus, les cyclones les plus violents du Pacifique sont enregistrés au cours des années El Niño.

\section{El Niño dans le passé}

Des documents historiques ont relaté l'occurrence d'El Niño dans le passé, ce qui prouve que ce phénomène naturel n'est pas récent. Nous disposons de l'enregistrement instrumental de la température, des précipitations, de la pression atmosphérique et des vents pendant les événements El Niño qui se sont produits depuis 1950. Ces enregistrements s'effectuent maintenant par satellites alors qu'auparavant les mesures se faisaient à l'aide d'instruments embarqués à bord de bateaux. De plus, les mesures faites alors dans l'océan Pacifique Sud sont assez rares, les enregistrements ne sont pas continus et ils comportent de longues périodes muettes, par exemple lors des deux guerres mondiales. Les températures ont parfois été mesurées dans certaines îles, comme à Tahiti, depuis la fin du $\mathrm{XIX}^{\mathrm{e}}$ siècle jusqu'au milieu du $\mathrm{xx}^{\mathrm{e}}$ siècle, mais ces mesures ont rarement été effectuées en un même site et présentent souvent des lacunes plus ou moins longues. Les relevés fournis par les dossiers maritimes des grands ports de la région ont été attentivement dépouillés et ont révélé qu'El Niño s'était manifesté au cours des derniers siècles de façon presque périodique, tous les deux à cinq ou sept ans. C'est vers 1920 que Walker, un océanographe britannique basé en Inde, intrigué par cette dynamique climatique, a cherché à en caractériser et à en quantifier les manifestations. Il a rassemblé et comparé, dans ce but, toutes les mesures (pression atmosphérique, précipitations, température) susceptibles d'avoir été réalisées de part et d'autre de l'océan et sur des îles parsemant le Pacifique intertropical. Il s'est alors avéré que le paramètre reproduisant le plus fidèlement la succession des événements El Niño au cours du temps était la différence de pression atmosphérique entre Tahiti (en Polynésie française) et Darwin (ville située au nord de l'Australie). Cette différence est devenue un index de référence. Bien que la mesure de la pression atmosphérique soit une des mesures physiques parmi les plus fiables (elle l'est depuis le début du siècle dernier), la confiance dans cet index est cependant restée limitée. L'enregistrement de paramètres plus fiables, basés, par exemple, sur la variation de la température de l'eau océanique dans des limites spatiales bien définies est désormais utilisé. L'examen d'un indice récemment retenu, l'index «El Niño 3 » (figure 5) permet d'observer que, depuis 1975 environ, les périodes El Niño, marquées par une hausse de température le long de l'Équateur et des côtes américaines, sont plus nombreuses que les périodes caractérisées, dans les mêmes régions, par les baisses de températures résultant de l'ascension d'eaux profondes, et donc symptomatiques de La Niña. Avant 1975, la situation était inverse : la Niña se faisait plus fréquente. De plus, d'autres modifications des conditions océaniques comme atmosphériques se sont produites simultanément plus au nord ou plus au sud de l'océan Pacifique 7 . Que s'est-il passé avant 1975? La simultanéité de ces changements est-elle une coïncidence? De tels épisodes couvrant plusieurs décennies se sont-ils déjà produits par le passé ? Il est difficile de répondre à ces questions. Les index cités ci-dessus ne suffisent pas pour donner une information précise sur les variations climatiques dans les deux hémisphères, à la fois dans le temps et dans l'espace. Les climatologues et les océanographes aimeraient disposer de séries de données sur des modifications océaniques et atmosphériques passées, précises et bien

7. Ces événements sont connus sous le nom de PDO (Pacific Decadal Oscillation) dans le nord de l'océan Pacifique. 


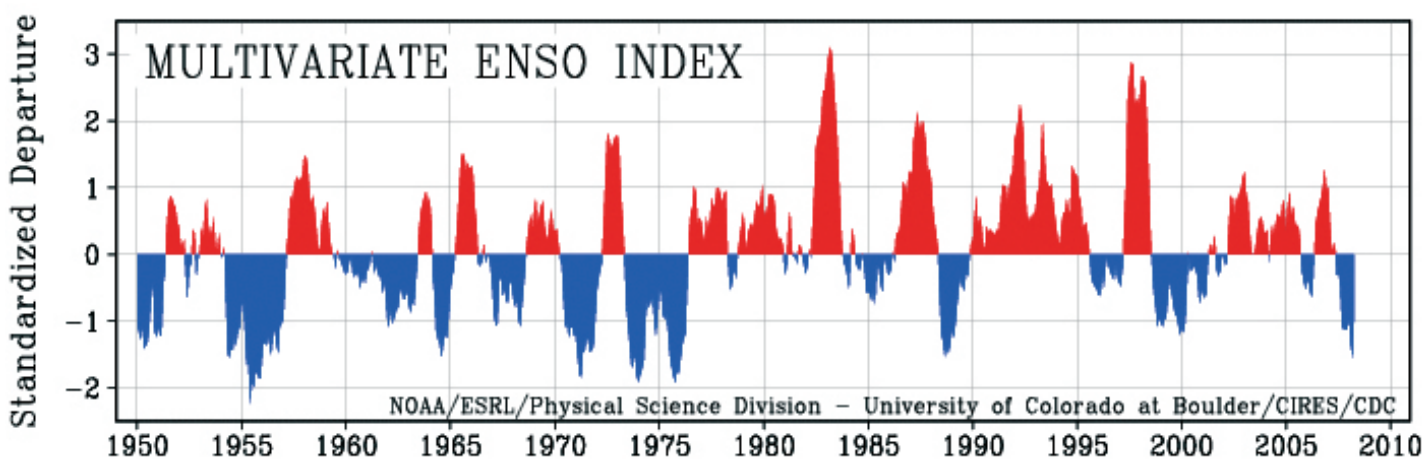

Figure 5. - Les index usuels appliqués à l'identification d'El Nino prennent en compte des paramètres divers, tels que la température, l'intensité du vent, la nébulosité, la hauteur du niveau marin. El Nino correspond, sur ce diagramme, aux pics positifs, la Nina aux pics négatifs. La situation climatique a changé en 1975 : les événements El Nino ont été relativement moins fréquents avant 1975 qu'ils ne le sont devenus après ( http://www.cdc.noaa.gov/ENSO)

datées, afin de les comparer à des mesures de mêmes natures réalisées dans des environnements actuels.

\section{Les indicateurs paléoclimatiques}

\section{Généralités}

Au milieu du siècle dernier les spécialistes du climat du passé utilisaient des traceurs indirects qui témoignent des conditions de température dans des temps plus ou moins reculés. Le support le plus souvent utilisé, au sein de sédiments marins, était constitué de minuscules squelettes calcaires de petits organismes, comme les foraminifères. La reconnaissance de la présence simultanée de plusieurs organismes de cette famille ayant vécu dans des conditions de température ou de salinité semblables fournissait une information, qualitative, sur ces paramètres environnementaux. Mais, au-delà d'approximations qualitatives, la température et/ou la salinité peuvent désormais être quantifiées précisément à partir de mesures physiques effectuées sur le squelette de ces foraminifères. Les mesures les plus pratiquées reposent sur celle du rapport des quantités des deux isotopes de l'oxygène, déjà évoqués. La transcription de données isotopiques en données climatiques (essentiellement de température) nécessite, au préalable, l'établissement de formules de passage entre les valeurs isotopiques mesurées et la valeur « climatique » recherchée. C'est ce que nous appelons la calibration, dont la précision conditionne celle des reconstitutions. Le plus souvent, ces formules de calibration sont obtenues en mesurant le rapport isotopique de l'oxygène dans des organismes ayant connu des conditions de croissance soi- gneusement consignées, soit dans la nature, soit en culture.

Mais les résultats les plus précieux ont été obtenus dans les glaces. Les molécules d'eau constituant la glace fournissent, à travers la valeur du rapport isotopique de leurs atomes d'oxygène (et d'hydrogène), des données susceptibles d'être transcrites en termes climatiques, essentiellement thermiques. De même les inclusions gazeuses $\left(\mathrm{CO}_{2}\right.$, par exemple) ou solides (cendres volcaniques ou particules de loess éoliens) présentes au sein de la glace fournissentelles également des données, respectivement quantitatives $\left(\mathrm{CO}_{2}\right)$ et qualitatives (particules éoliennes) sur l'histoire climatique de l'environnement. C'est ainsi qu'a pu être reconstituée la variation périodique de la température atmosphérique, jusqu'aux domaines polaires, pendant le Quaternaire récent, et sa parfaite correspondance avec la variation de la température des eaux océaniques, déduite de l'analyse isotopique de tests de foraminifères. L'analyse de l'air contenu dans la glace a, quant à elle, permis de découvrir que la concentration atmosphérique en gaz carbonique avait varié, elle aussi, dans le même temps et dans le même sens que la température atmosphérique. Ainsi la succession des grandes périodes glaciaires ayant affecté les océans et les continents, au cours du dernier million d'années, selon plusieurs périodicités, a-t-elle pu être reconstituée. Une découverte majeure éclairant la paléoclimatologie du Quaternaire a couronné tous ces résultats locaux ou plus globaux, celle du mécanisme général ayant été à l'origine de ces variations climatiques périodiques, à l'échelle de quelques dizaines ou de quelques centaines de milliers d'années. $\mathrm{Ce}$ mécanisme est astronomique et résulte de l'influence, sur le climat terrestre, de la position 
relative, toujours changeante, de la terre et du soleil.

\section{L'application du thermomètre isotopique aux coraux: une méthode en progrès}

La méthode isotopique a donc été appliquée à des squelettes de coraux. Il y a plus d'un quart de siècle, en 1972, deux chercheurs américains, Weber et Woodhead (1972), après avoir prélevé des fragments de coraux d'espèces variées au large d'îles tropicales, ont pu confirmer que la quantité relative des isotopes de l'oxygène dans leur squelette dépendait de la température de l'eau de mer dans laquelle ils s'étaient développés.

Mais il est apparu que chaque espèce présentait une calibration propre, c'est-à-dire que la loi de calibration déterminée pour cette espèce ne s'appliquait qu'à elle seule ou, en d'autres termes, que chaque espèce nécessitait l'écriture de sa propre loi de calibration. Les chercheurs considérèrent que la variété de ces lois de calibration était due à l'origine biologique, vivante, de l'animal dont le code génétique induirait, dans chaque espèce, un fractionnement spécifique des isotopes de l'oxygène au cours de la cristallogenèse. Mais cette contrainte n'entraîna pas de difficulté majeure dans l'application de la méthode isotopique à des espèces bien déterminées. La réponse des chercheurs fut la même lorsque se présentèrent des cas où des colonies de même espèce, développées dans les mêmes conditions de température, révélèrent des rapports isotopiques différents. La méthode consista alors à calibrer la relation températurerapport isotopique de l'oxygène sur des couches d'accroissement récentes dans lesquelles la température de croissance avait pu être mesurée avec précision, mensuellement au cours du temps.

Les difficultés précédentes ont eu le mérite de conférer un rebond à l'exploitation de la méthode isotopique. L'interprétation générale des différences apparaissant entre des espèces proches fut qu'elles résultaient d'un effet « vital » ou « biologique » intervenant dans la cristallogenèse. Les lois du fractionnement isotopique de l'oxygène dans de l'aragonite précipitant dans des conditions purement minérales, apparaissaient transgressées dans ces tissus biologiques. Elles l'étaient, vraisemblablement, au contact - ou par - les constituants organiques (biologiques stricto sensu) présents dans le sque- lette corallien. Cette hypothèse est débattue actuellement et nous la re-évoquerons dans le paragraphe « La biominéralisation».

C'est donc au prix de multiples précautions qu'est appliquée, avec succès, la méthode isotopique dite de l'oxygène aux squelettes coralliens et à ceux d'autres organismes. L'utilisation du corail, en terme d'enregistreur du climat, a connu un réel regain d'intérêt après 1989, année pendant laquelle des travaux nouveaux confirmèrent la relation étroite existant entre la température et le rapport isotopique de l'oxygène, au fur et à mesure de la formation du squelette, c'est-à-dire les résultats de Weber et Woodhead (1972). L'on utilise, de préférence, des coraux massifs semblables à celui de la figure 2. Le prélèvement se fait sous l'eau, à l'aide d'un « carottier $»^{8}$ manipulé par des plongeurs. On recueille ainsi un cylindre d'aragonite, une "carotte". Celle-ci est ensuite découpée en lames de quelques dizaines de millimètres d'épaisseur, avec un pas d'échantillonnage ${ }^{9}$ aussi réduit que possible. Les plaques sont alors passées aux rayons $\mathrm{X}$ dans une machine apparentée à celle utilisée pour obtenir des radiographies médicales. Les photographies révèlent toute l'histoire de la formation du squelette, par exemple les arrêts connus par sa croissance ou les dégâts que lui ont infligés les animaux foreurs. Sur la radiographie, l'orientation d'un polype est soulignée par un petit segment clair correspondant à l'emplacement de son sac gastrique (figure 1). Un alignement de polypes suivant un même rayon apparaît alors sous la forme d'un segment, clair, plus ou moins long, plus ou moins continu. Nous tentons alors de suivre la même succession de polypes sur la plus grande distance possible, dans la direction de croissance maximum. La précision du thermomètre est estimée à $0,6^{\circ} \mathrm{C}$. Il présente le fantastique avantage d'être quasiment continu dans le temps.

Dans la mesure où le rapport isotopique de l'oxygène dans le squelette dépend aussi $\mathrm{du}$ rapport isotopique de l'oxygène dans l'eau de mer - qui varie lui-même comme la salinité et se révèle donc sensible aux précipitations, à l'évaporation et aux mélanges de masses d'eau il convient de contourner cet inconvénient en utilisant un autre couple chimique, le couple strontium ${ }^{10}$ calcium, à travers le rapport $\mathrm{Sr} / \mathrm{Ca}$. Ce dernier est considéré comme un indicateur de la température. La formule donnant la température en fonction de la valeur de ce rapport est établie de la même façon que celle reliant la

8. Un cylindre d'acier d'environ 6 à $8 \mathrm{~cm}$ de diamètre.

9. Mesuré par la différence d'âges des échantillons aux deux extrémités de la plaquette.

10. Un élément pouvant se substituer au calcium dans le carbonate de calcium. 
température au rapport des isotopes de l'oxygène, c'est-à-dire de façon empirique. Ainsi l'ensemble de ces indicateurs permettent-ils de reconstituer la température et la salinité, deux paramètres essentiels dans la reconstitution de conditions océanographiques.

\section{La biominéralisation}

L'exploitation de la méthode isotopique de l'oxygène dans les coraux a connu, récemment, un progrès dont les conséquences ne sont pas encore totalement mesurées.

Malgré les signes selon lesquels des «effets vitaux » étaient à même de jouer des rôles spécifiques dans le fractionnement isotopique de l'oxygène dans les squelettes coralliens, les géochimistes ont trop oublié, durant les dernières décennies, que le squelette corallien est constitué d' un minéral déposé par un organisme vivant. Il ne viendrait à l'idée de personne de penser que les os humains sont de simples dépôts de phosphate de calcium purement minéral. La matière organique est un constituant essentiel de nos os. Ainsi en est-il du squelette des coraux. L'observation du squelette corallien à très petite échelle, au microscope électronique, y a fait apparaître une complexité qui avait totalement échappé aux géochimistes: les unités minérales se présentent sous de multiples formes cristallines, témoignant de modes de croissance variés et, en outre, de la matière organique apparaît intimement liée à ces cristaux. L'organisation de ceux-ci au sein du squelette (figure 6) est remarquable et laisse peu de doute sur l'existence de contrôles d'origine biologique au cours de leur élaboration. Ces observations se reproduisent aux plus petites échelles (figure 6). Nous avons encore beaucoup à apprendre de l'étude de tels biomatériaux. Ils constituent des éléments robustes, souvent fabriqués à faible coût énergétique. Leur étude constitue l'une des branches les plus prometteuses des nanotechnologies.

Pour reconnaître les paramètres physiques comme la température ou la salinité - qui sont enregistrés dans le squelette corallien, nous devons donc savoir faire la part des réponses de natures respectivement biologiques et minérales apportées par le corail aux différents facteurs externes.

\section{L'importance des cultures en milieu contrôlé}

Il est évidemment difficile d'examiner, sur le terrain, les effets respectifs de la lumière et de la température sur la croissance d'une colonie corallienne, leur augmentation allant souvent de pair. Le meilleur moyen pour étudier l'influence de la lumière sur les coraux, sans modifier la température, est de les cultiver. Le CSM (Centre scientifique de Monaco), situé à proximité de l'Aquarium de Monaco, s'est spécialisé dans la culture de coraux dans des milieux contrôlés. Ses chercheurs ont ainsi réussi à bouturer une branche de corail comme on le fait de celle d'une plante. Le fragment prélevé, collé sur une plaque de verre et placé dans un aquarium, est capable de former à nouveau du squelette, à la fois sur le fragment bouturé, mais aussi sur la plaque de verre. Ce sont des assemblages minéraux comme ceux-là que nous cherchons à analyser car nous avons la certitude qu'ils se sont constitués dans des conditions connues (figure 7). Il en est cependant des coraux comme des plantes, cette culture nécessite des soins particuliers que seuls les spécialistes savent prodiguer.

La collaboration des biologistes est indispensable à cette approche géochimique car nous devons savoir associer les changements biologiques subis par l'animal au cours de son développement et les mesures géochimiques que nous pratiquons. La contribution des biologistes est, en particulier, indispensable pour contrôler les échanges qui s'opèrent entre l'animal et l'algue qu'il abrite, à l'échelle des xanthelles ellesmêmes, mais aussi - et surtout - à celle des produits de leur métabolisme.

\section{Les mesures faites à petite échelle}

Grâce à des appareils dont il n'existe que peu d'exemplaires dans la communauté scientifique française, nous pouvons effectuer les mesures du rapport des quantités des isotopes stables de l'oxygène et du rapport $\mathrm{Sr} / \mathrm{Ca}$ sur de très petits échantillons. L'opération nécessite un soin tout particulier: dans un premier temps les différents composants, minéraux et organiques, sont repérés au microscope de façon à effectuer des mesures parfaitement ciblées. Cette étape est réalisée en collaboration avec des experts de la biominéralisation de l'université d'Orsay. Les mesures elles-mêmes sont effectuées dans d'autres laboratoires, à Nancy ou au Muséum national d'Histoire naturelle. Outre les mesures géochimiques précédemment citées - rapports isotopiques de l'oxygène et rapport $\mathrm{Sr} / \mathrm{Ca}-$, la compréhension du mode de dépôt demande l'analyse de composés chimiques, notamment organiques, qui ne peut être réalisée qu'à l'aide d'instruments per- 


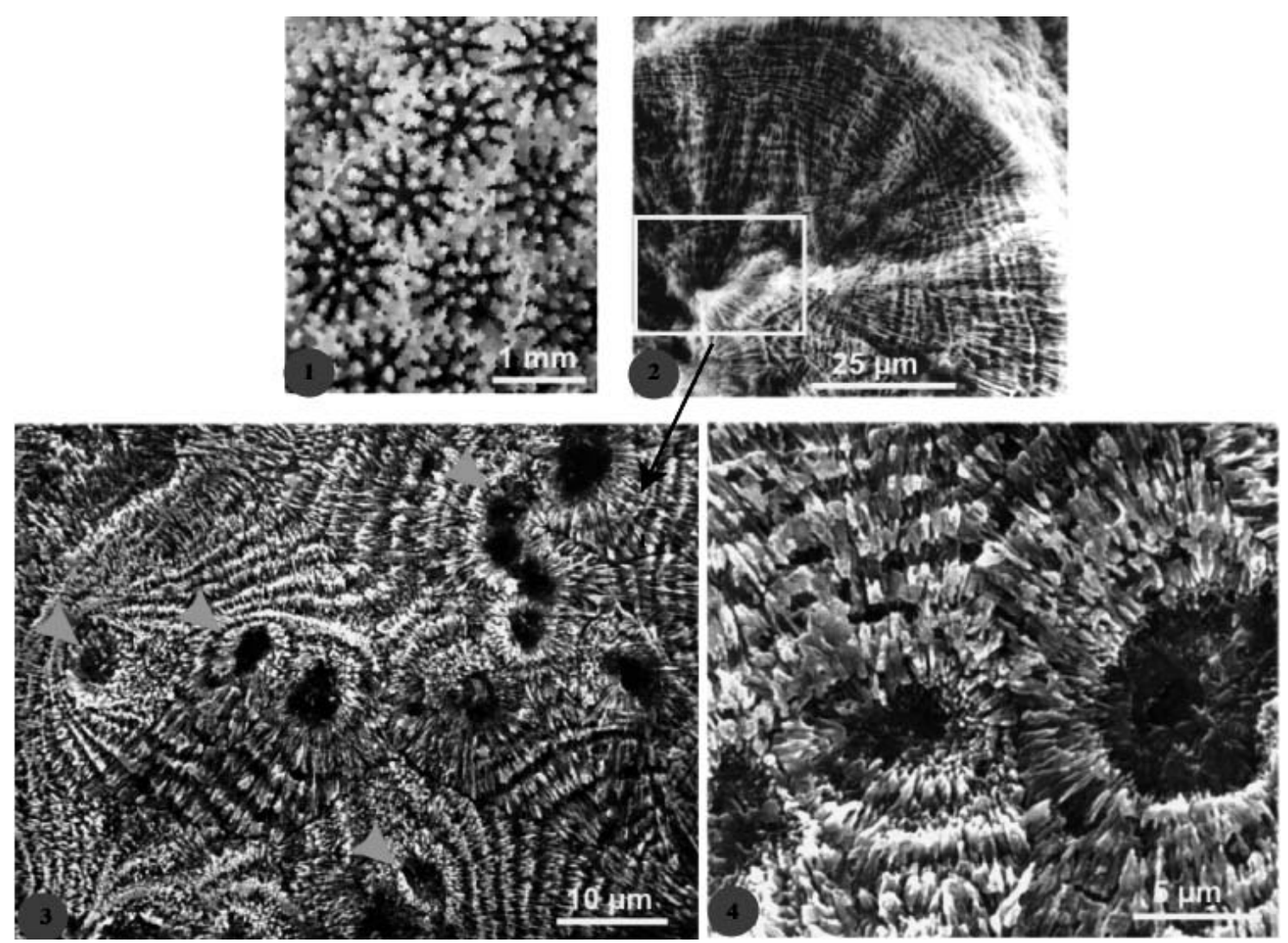

FIgURE 6. - Structures observables au sein du squelette de Porites (Photo : J.-P. Cuif)

6.1. Comme toutes les espèces de coraux, Porites possède un squelette très orné. Plus la zone observée est restreinte $(2,3,4)$, plus l'ornementation est développée.

6.3. et 6.4. montrent des trous ayant résulté d'une dissolution de l'aragonite superficielle sous l'effet d'une attaque ménagée destinée à faire apparaître les structures du squelette. Les couches concentriques sont bien visibles.

formants comme le synchrotron ${ }^{11}$. Les résultats obtenus permettent, en combinant les différentes mesures que nous sommes capables d'effectuer à cette petite échelle, de déceler des mécanismes physiques et chimiques mis en œuvre lors de l'élaboration du squelette et, tout particulièrement, des mécanismes organo-minéraux où les constituants de la matière organique prennent une part active dans la formation des entités minérales. Ces investigations, qui en sont à leurs débuts, ne répondront certainement pas à toutes les questions que nous nous posons encore. Néanmoins, elles nous apportent, dès à présent, des éléments sur ce qui était attribué - sans preuve formelle - à des effets biologiques, " vitaux ». Nous sommes maintenant capables de distinguer, dans les signaux obtenus, la part d'activités biologiques de celle qui ne dépend que du milieu externe, c'est-à-dire de celles que nous cherchons à reconstituer à travers la composition isotopique.
Nous savons maintenant que la lecture du message que nous cherchions à décrypter était et demeure toujours - difficile à cause de l'interférence des processus inorganiques et de processus organiques (biologiques). Pendant longtemps l'influence de la biologie a été considérée comme suffisamment faible pour être négligée alors que nous nous apercevons maintenant que les valeurs des mesures que nous considérions comme des traceurs climatiques fidèles perdent partiellement de leur précision sous l'effet du mélange de leur signal avec celui - ou ceux - de tels effets biologiques.

Prenons l'exemple d'une hausse de la température de l'eau de mer. Les conséquences de celle-ci vont se manifester de multiples façons. En été l'animal, appréciant des températures supérieures à celles de l'hiver, verra son métabolisme et, notamment, sa respiration augmenter. Les algues qui vivent en symbiose avec lui réagissent de même, et leur respiration et leur activité 

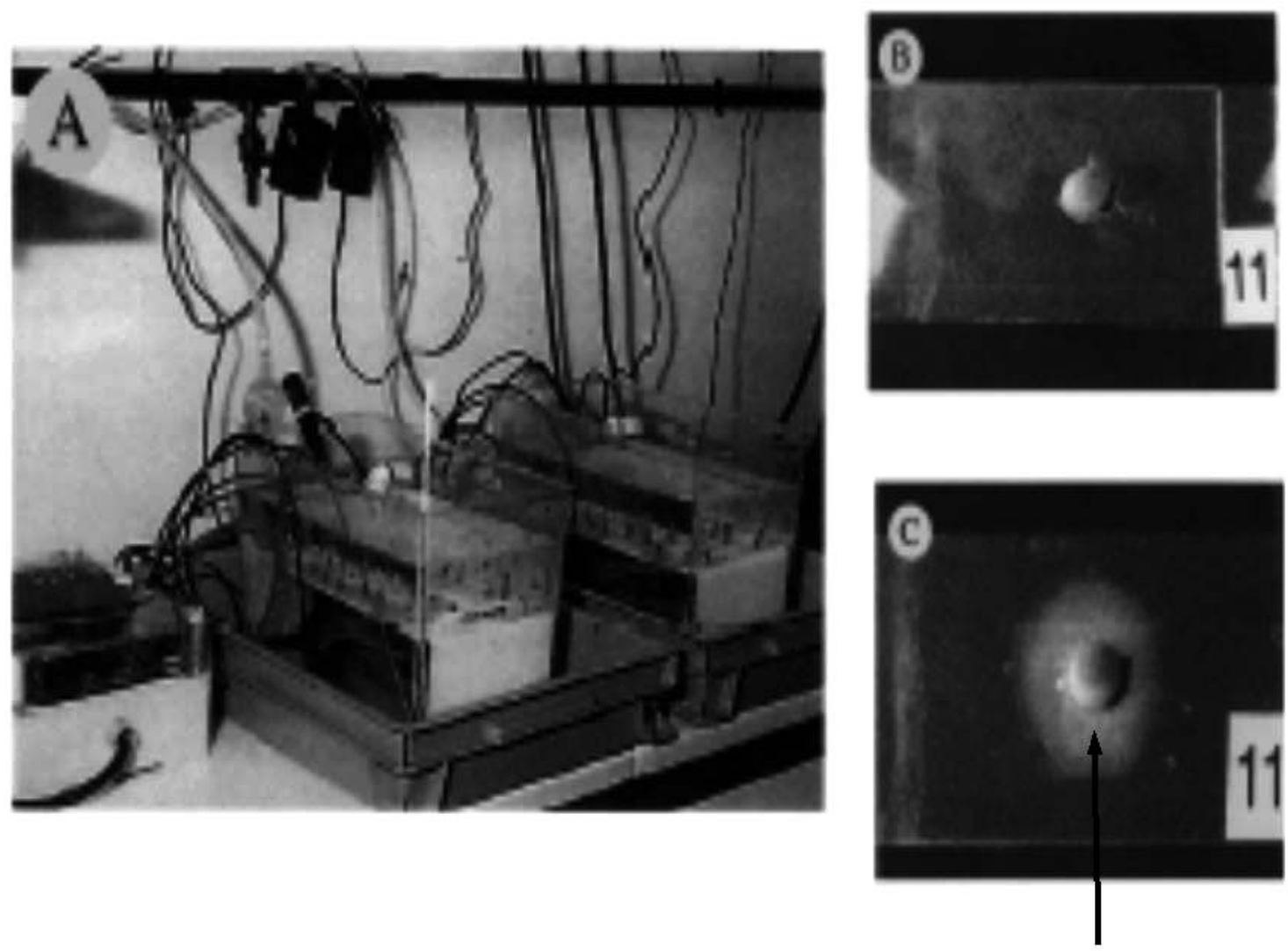

corail néoformé

Figure 7. - Cultures de coraux au Centre scientifique de Monaco (Photo : S. Reynaud)

A. - Aquariums dans lesquels les paramètres de croissance sont contrôlés (température, intensité lumineuse, salinité...).

$\mathrm{B}$ et C. - Des petits rubans de coraux sont collés sur des lames de verre à la surface desquelles les coraux développent un nouveau squelette.

photosynthétique s'intensifient. Chacune de ces variations métaboliques s'inscrit, d'une manière propre à chaque espèce, dans la valeur du rapport isotopique de l'oxygène du carbonate $\mathrm{du}$ squelette de cette espèce duquel nous cherchons à extraire une valeur de température. Chacune de ces interférences contribuera à un bruit de fond qui masque l'effet purement physico-chimique recherché.

\section{Le corail, véritable archive de l'océan tropical}

Nous avons donc la capacité de reconstituer des variations de température et, à travers elles, des processus océanographiques comme des courants ou des remontées d'eaux froides, telles que celles qui sont associées à El Niño. En outre, nous connaissons aussi les fluctuations de la salinité qui dépendent des mélanges de masses d'eaux, donc des courants marins, des précipitations ou de l'évaporation, potentiellement inten- ses l'une et l'autre dans cette région du monde. Nous pouvons comparer ces valeurs avec des données historiques recueillies dans les journaux de bord de bateaux ou dans les ports de la côte sud-américaine où sont archivés, de façon ponctuelle, les événements El Niño au cours du temps. La prise en compte de séries de valeurs provenant de diverses colonies coralliennes, choisies dans des sites permettant de discerner au mieux les différentes manifestations environnementales, sera nécessaire pour obtenir une vision régionale, puis globale, des anomalies climatiques passées. Il sera possible d'observer, en particulier, quelles ont été les éventuelles modifications d'El Niño au cours de périodes comme le Petit Âge Glaciaire (connu pour avoir été une période plus froide que l'actuelle, d'environ $1^{\circ} \mathrm{C}$, entre le $\mathrm{XV}^{\mathrm{e}}-\mathrm{XVI}^{\mathrm{e}}$ et le $\mathrm{XIX}^{\mathrm{e}}$ siècle) ou durant l'Optimum du Moyen Âge (caractérisé par des températures semblables aux températures actuelles). Ces événements sont-ils restés restreints au pourtour de l'Atlantique ou 


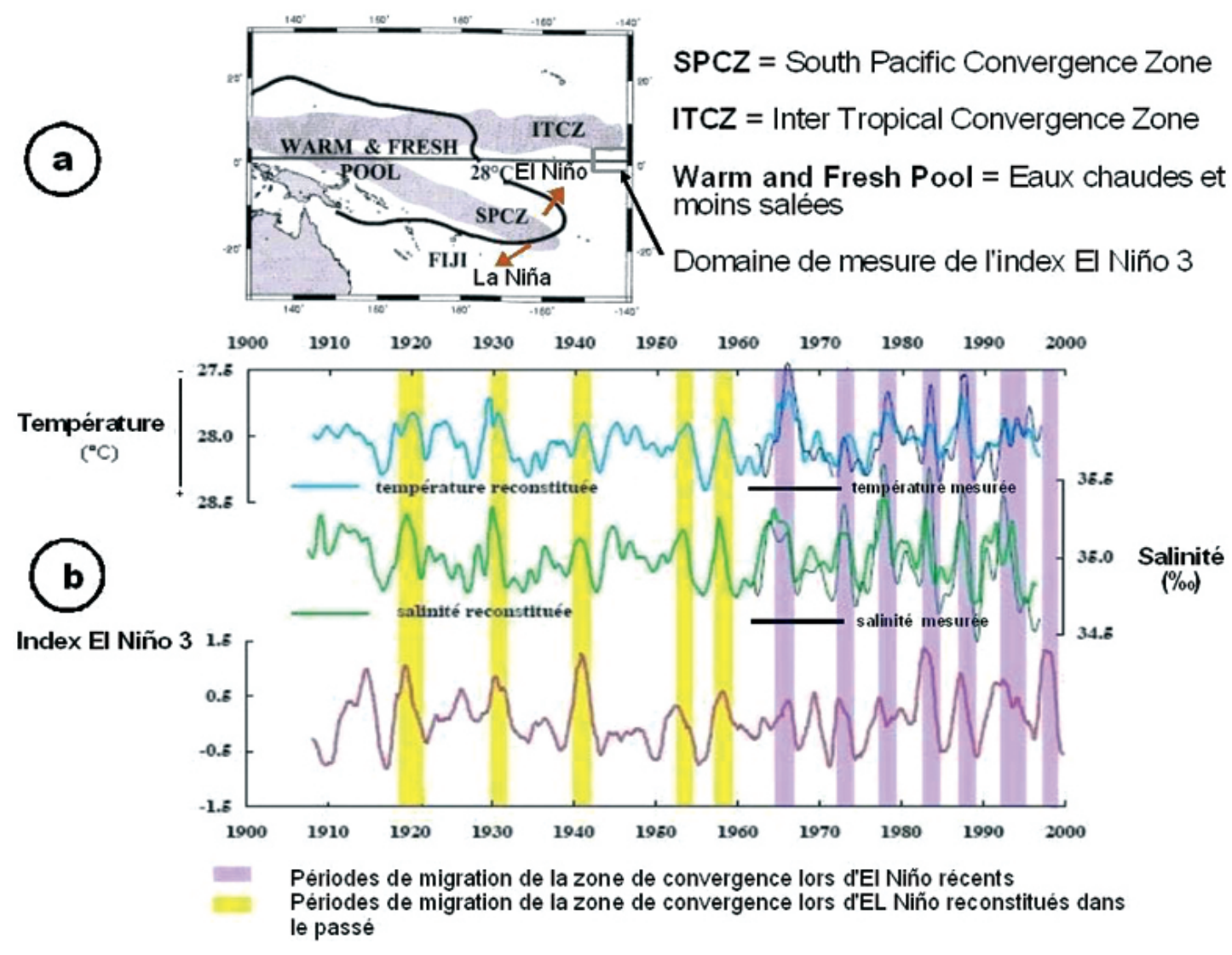

FIGURE 8. - Dynamique océanique et climatique dans le domaine océanique entourant Fiji. Températures et salinités de l'eau marine superficielle reconstituées d'après la composition isotopique d'un squelette corallien, carotté à Fiji. Des nuages abondants se forment dans la zone de convergence intertropicale (ITCZ) et la zone de convergence du Pacifique Sud (SPCZ). L'archipel de Fiji est situé dans la zone la plus chaude de l'océan Pacifique, au droit de la SPCZ, donc dans une région parfaitement adaptée pour observer les migrations de cette zone SPCZ. Pendant une période normale, la zone SPCZ est située là où elle est représentée sur la carte. En période d'El Nino, cette zone migre vers le Nord et entraine, à Fiji, une diminution des précipitations, une augmentation de la salinité et une diminution de la température. La reconstitution de la température marine superficielle (Sea Surface Temperature, SsT) est le résultat d'un traitement statistique de sept séries de données, celle de la salinité marine superficielle (Sea Surface Salinity, sss) d'un traitement de quatre séries. Leurs résultats sont à comparer avec des mesures instrumentales réalisées de 1960 à 1997 et avec l'index « El Nino 3 », basé sur la mesure de la température marine superficielle le long d'un segment de l'équateur dans le Pacifique oriental (voir la carte, fig. 8a). L'amplitude des variations de la température et de la salinité ne dépasse pas $1{ }^{\circ} \mathrm{C}$ pour la température et $1 \%$ pour la salinité. Des baisses de température et des augmentations de salinité ont accompagné les évènements El Niño depuis 1950 ainsi que les évènements signalés par l'index El Niño 3 (fig. 8b). La fréquence des migrations de la zone SPCZ a clairement augmenté depuis 1975 (voir cahier couleur après p. 144)

leurs effets ont-t-ils été ressentis à l'échelle du globe?

Nous en sommes à ce jour au début de l'exploitation des archives coralliennes. Nous savons maintenant reconstituer le passage d'une zone fortement pluvieuse au-dessus de la zone la plus chaude de l'océan Pacifique lors d'un épisode El Niño et déterminer, de façon indépendante, les variations de température de l'eau de surface de l'océan dans la même zone (fig. 8), avec une précision qui se rapproche de celle d'un thermomètre.

La méthode peut être encore améliorée. Nous allons continuer à examiner les effets spéci- fiques de chaque facteur externe, température, lumière, ou salinité, grâce aux croissances obtenues en cultures, à très petite échelle spatiale. L'on attend de l'utilisation conjointe des outils précédemment cités la possibilité de développer de nouveaux enregistreurs, en particulier de l'ensoleillement.

Maintenant que nous connaissons mieux comment sont enregistrés les changements climatiques, nous pouvons envisager d'utiliser des coraux toujours plus anciens. Ceux-ci ont déjà contribué à préciser la fiabilité de datations effectuées sur des matériaux d'âge quaternaire avec des méthodes chronologiques spécifiques $\left({ }^{14} \mathrm{C}\right.$, 
notamment) et à décrire avec une bonne précision la remontée des niveaux marins pendant les dernières déglaciations. Il existe, en effet, dans les zones tropicales des vestiges de terrasses construites par les coraux, témoins de récifs anciens et qui sont autant de repères de niveaux marins temporairement stabilisés dans le passé. La croissance des coraux n'a cessé de réagir aux changements du niveau marin au cours de la fonte ou de la formation des glaces polaires au cours des dernières centaines de milliers d'années. La datation de ce matériel est possible, à condition de s'assurer de sa bonne conservation au cours du temps. Cette précaution permet d'éviter de dater, à la place d'un échantillon de corail sain et donc fiable, un échantillon mixte associant, par exemple, un fragment de corail et un fragment de calcaire inorganique ayant précipité à son contact. Les coraux seuls peuvent fournir des informations encore indisponibles, par exemple sur les saisons et les variations climatiques à l'échelle de quelques dizaines d'années. Ainsi, pouvons-nous espérer quantifier des facteurs climatiques, au cours de périodes de temps restreintes, avec une précision jamais atteinte.

Le changement climatique auquel nous assistons actuellement met en péril les récifs coralliens. En sont essentiellement responsables les augmentations de la teneur en gaz carbonique dans l'atmosphère et de la température. Les effets de cette dernière se manifestent par le blanchissement du squelette et par l'augmentation de la vulnérabilité du corail aux attaques bactériennes. Les effets de l'acidification de l'océan, résul- tant de la dissolution, en son sein, du $\mathrm{CO}_{2}$ atmosphérique, sont plus délicats à évaluer. Deux réactions antagonistes sont, en effet, susceptibles de se produire. D'une part, le gaz carbonique ainsi disponible a un effet fertilisant sur les algues symbiontes du corail. Mais, d'autre part, l'excès de $\mathrm{CO}_{2}$ peut entraîner un ralentissement de la formation du squelette des coraux, qui pourrait conduire à la formation d'organismes mous. L'influence globale de ce facteur sur la symbiose corallienne est donc très complexe. Dans les années 1990, les Japonais avaient fondé de grands espoirs sur la possibilité du stockage de $\mathrm{CO}_{2}$ par les coraux. Les premiers aménagements avaient été ébauchés lorsque la preuve d'un effet global contraire fut démontrée. La question demeure maintenant de savoir si la construction de récifs coralliens est ralentie de façon notable dans les conditions actuelles et si même des récifs anciens ne seront pas menacés de dissolution. Et nous-mêmes combien de temps avons-nous encore pour lire dans le squelette des coraux les événements climatiques qu'ils ont connus?

\section{BIBLIOGRAPHIE}

Robin R., C. Petron et C. Rives, 1987. Les coraux, Nouvelle-Calédonie, Tahiti, Réunion, Antilles. Nouméa, Les Éditions du Pacifique, 144 p.

Weber J.N. and P.M.J. WoodheAd, 1972. Temperature dependence of oxygen-18 concentration in reef coral carbonates, Journal Geophys. Res. 77, pp. $463-$ 473. 
